\title{
Paratesticular Rhabdomyoma
}

National Cancer Institute

\section{Source}

National Cancer Institute. Paratesticular Rhabdomyoma. NCI Thesaurus. Code C162496.

A rhabdomyoma that arises from the paratesticular region. 